\title{
Ejercicio, bienestar psicológico, calidad de sueño y motivación situacional en estudiantes de educación física
}

\author{
Exercise, psychological well-being, sleep quality and \\ situational motivation in physical education students
}

\section{Exercício, bem-estar psicológico, a qualidade do sono e motivação situacional em estudantes de educação física}

\author{
E. Cervelló, F. Peruyero, C. Montero, D. González-Cutre, V.J. Beltrán-Carrillo y J.A. Moreno-Murcia
}

Centro de Investigación del Deporte. Universidad Miguel Hernández de Elche (España)

Resumen: El objetivo de este trabajo ha sido comprobar si la exposición a diferentes intensidades de actividad física aguda ocasionaba cambios en el bienestar psicológico (vitalidad subjetiva y estado afectivo), la calidad del sueño y la motivación intrínseca situacional en estudiantes adolescentes en clases de educación física. Para ello, 120 estudiantes ( 50 chicos y 70 chicas) con edades comprendidas entre los 16 y 20 años completaron, antes y después de pasar por cada situación experimental, medidas de estas variables. Los resultados mostraron, en primer lugar, que había asociaciones directas entre la vitalidad subjetiva, el estado afectivo positivo y la calidad del sueńo. En segundo lugar, se encontró un incremento significativo en la motivación intrínseca situacional y disminución en el estado afectivo negativo post-sesión al comparar las intensidades vigorosa y ligera. La mayoría de las dimensiones del bienestar psicológico mejoraban cuando se comparaban las situaciones pre intervención y post intervención, independientemente de la intensidad del ejercicio. Contrariamente a nuestras hipótesis, la calidad del sueño no mejoró al practicar actividad física. La principal conclusión es que la realización de actividad física aguda, independientemente de la intensidad del mismo, mejora el bienestar. Respecto a la motivación, la actividad física vigorosa es la que provoca mayores cambios motivacionales, debiendo tener este aspecto en cuenta a la hora de diseñar programas de actividad física para adolescentes. Palabras clave: Ejercicio, bienestar, calidad del sueńo, motivación.

Abstract: The aim of this study was to determine how the exposure to different intensities of acute physical activity, caused changes in psychological well-being (subjective vitality and affective state), sleep quality and situational intrinsic motivation in adolescent students on physical education classes. 120 students ( 50 boys and 70 girls) aged between 16 and 20 years, completed different questionnaires to measure these variables.

The results showed direct and positive associations between subjective vitality, positive affect state and the quality of sleep and significant differences, increasing situational intrinsic motivation and decreasing post-session negative affect when comparing light and vigorous intensities. Almost all dimensions of psychological well-being improved when comparing the preintervention and post intervention situations, regardless of physical activity intensity. Contrary to our hypothesis, sleep quality did not improve by practice of physical activity. The main conclusion is that the acute exercise, regardless of intensity, improves well-being and that vigorous physical activity is causing major motivational changes, must take this into account when designing physical activity programs for adolescents.

Keywords: Exercise, well-being, quality of sleep, motivation.

Resumo: O objectivo deste trabalho consistiu em comprovar se a exposição a diferentes intensidades de exercício agudo ocasionava alteraçôes no bem-estar psicológico (vitalidade subjectiva e estado afectivo), na qualidade de sono e na motivação intrínseca situacional de estudantes adolescentes em aulas de educação física. Para tal, 120 estudantes (50 rapazes e 70 raparigas) com idades compreendidas entre os 16 e os 20 anos preencheram, antes e depois de passarem por cada situação experimental, instrumentos de medida destas variáveis. Os resultados mostraram, em primeiro lugar, que havia associaçóes directas entre a vitalidade subjectiva, o estado afectivo positivo e a qualidade de sono. Em segundo lugar, verificou-se um incremento significativo na motivação intrínseca situacional e uma diminuição do estado afectivo negativo após a sessáo, ao comparar as intensidades vigorosa e ligeira. A maioria das dimensôes do bem-estar psicológico melhorou quando comparadas as situaçôes pré-intervenção e pós-intervenção, independentemente da intensidade do exercício. Contrariamente às nossas hipóteses, a qualidade de sono não melhorou ao praticar actividade física. A principal conclusão é a de a realização de exercício agudo, independentemente da sua intensidade, melhora o bem-estar. Relativamente à motivação, a actividade física vigorosa é a que provoca maiores alteraçôes motivacionais, devendo ter-se este aspecto em consideração quando forem desenhados programas de actividade física para adolescentes.

Palavras-chave: Exercício, bem-estar, qualidade de sono, motivação.

\section{Introducción}

El ejercicio físico, cuando se practica de forma regular, es muy beneficioso para la salud y para el bienestar tanto físi-

\footnotetext{
Dirección para correspondencia [Correspodence address]: Eduardo M. Cervelló Gimeno. Centro de Investigación del Deporte. Universidad Miguel Hernández de Elche. Av / Universidad s/n. 03202 Elche, Alicante (España). E-mail: ecervello@umh.es
}

co como psicológico (Redd y Ones, 2006; Texeira, Carraça, Markland, Silva, y Ryan, 2012). Sin embargo los datos recientes indican que son pocas las personas que practican deporte de forma regular. De hecho, en Europa, un 42\% de la población, nunca practica actividad física (Eurobarómetro, 2013). Estos datos muestran una tendencia preocupante, pues 
en los últimos 5 años, se ha incrementado un 3\% el porcentaje de personas que nunca practican deporte o actividad física (Texeira et al., 2012).

A este respecto, una de las cuestiones que más preocupa a los investigadores es analizar las razones por las cuales las personas, aún sabiendo de los beneficios de la actividad física, no practican deporte (Redd y Ones, 2006).

Diferentes trabajos han intentado acotar los diferentes tipos de beneficios psicológicos que se obtienen de la práctica de actividad física (para una revisión ver Redd y Ones, 2006) y se ha encontrado que los cambios afectivos que se provocan durante el ejercicio pueden estar muy relacionados con la adherencia al mismo (Thayer, 1996). Igualmente, cuestiones tales como la intensidad del ejercicio, la duración del mismo y el tiempo que transcurre entre sesiones parecen estar relacionados con el bienestar percibido por parte de los practicantes.

Sin embargo, a pesar de que existe mucha literatura acerca del efecto de la práctica habitual de ejercicio sobre la mejora del bienestar y la adherencia a la práctica de actividad física (Gunnell, Crocker, Mack, Wilson, y Zumbo, 2014), no conocemos ningún trabajo que analice simultáneamente cómo la práctica aguda de actividad física afecta al bienestar psicológico y a la motivación situacional para la práctica de actividad física.

Recientes trabajos que han analizado bienestar y motivación en práctica deportiva (Ryan, Huta, y Deci, 2013) consideran que el bienestar cabe estudiarlo desde dos perspectivas: una denominada hedónica, en la que se considera el bienestar como la presencia de afecto positivo y ausencia de afecto negativo, y una segunda denominada eudaimónica, en la que el bienestar no consiste únicamente en la maximización de experiencias positivas y reducción de experiencias negativas, sino que se refiere a vivir de forma plena o dar realización a los potenciales humanos más valiosos (Ryan, Huta, y Deci, 2008). Estos trabajos se fundamentan en la idea de que la realización de conductas de búsqueda de la salud y el bienestar se basa en los niveles de autodeterminación de los indivíduos (Ryan y Deci, 2000). Así, esta teoría de la autodeterminación, indica que el bienestar es fundamentalmente consecuencia de un funcionamiento psicológico óptimo, y que este funcionamiento psicológico óptimo implica una adecuada satisfacción de diferentes tipos de necesidades básicas (competencia, autonomía y relaciones sociales). Por otra parte, también considera que el bienestar está estrechamente relacionado con la adopción de ciertas metas que permitan la aparición del mismo. Así, estas metas deben ser preferiblemente más intrínsecas que extrínsecas o desmotivadas y coherentes con los valores e intereses del individuo (Deci y Ryan, 2000; Vazquez, Hervás, Rahona, y Gómez, 2009).

Otra idea clave relacionada con los niveles de motivación y que recoge el Modelo Jerárquico de Motivación de Vallerand, $(1997,2001)$ es que ésta actúa a diferentes niveles de generalidad. Así se puede distinguir entre motivación global, contextual y situacional. Desde el punto de vista situacional la revisión efectuada por Vallerand (2007) indica que la motivación intrínseca situacional se ha relacionado con diferentes consecuencias psicológicas deseables. Entre éstas se incluyen: el flow situacional, la vitalidad, el afecto positivo, el disfrute, la satisfacción, el interés, concentración, esfuerzo, persistencia, y la adherencia al ejercicio. Y negativamente con la ansiedad, el agotamiento, el aburrimiento y la fatiga.

Por último, comentar que otro indicador que recientemente se ha asociado al bienestar, la salud y la calidad de vida, es la calidad del sueño (Polo-Kantola et al., 2014). De hecho, en un estudio reciente realizado con adolescentes, se ha encontrado que la cantidad y calidad del sueńo, se relaciona con el estado de ánimo, la depresión, la ansiedad y la autoestima, de forma que aquellos sujetos con menor cantidad y calidad de sueño, presentaban emociones más negativas frente a aquellos que tenían una mejor cantidad y calidad del sueño (Wong et al., 2013).

Así, el objetivo de este trabajo gira en dos direcciones. En primer lugar y desde una perspectiva correlacional, queremos analizar cómo se relacionan las diferentes variables de bienestar analizadas en este trabajo. En segundo lugar, y desde una perspectiva experimental, queremos comprobar si la exposición a diferentes intensidades de actividad física aguda (ligera, moderada o vigorosa), provocan cambios en el bienestar (estado afectivo y vitalidad), la calidad del sueño y la motivación intrínseca situacional.

Nuestra primera hipótesis, basada en estudios que han analizado la relación del estado afectivo y la calidad del sueño (Polo-Kantola et al., 2014), es que habrá una relación positiva entre bienestar psicológico (vitalidad y estado afectivo positivo) y calidad del sueño y negativa entre estado afectivo negativo y calidad del sueño.

Nuestra segunda hipótesis basada en los estudios que han analizado el efecto del ejercicio habitual en el bienestar, (Teixeira et al., 2012) es que la sesión de ejercicio vigorosa provocará una mayor mejora del bienestar, calidad del sueño y motivación intrínseca situacional que las sesiones ligeras o moderadas. También creemos, a la vista de los trabajos realizados sobre efecto agudo de la actividad física (Reed y Ones, 2006), que todos los tipos de actividad física aguda provocarán cambios positivos en bienestar, y calidad del sueño frente a la situación de no actividad física.

\section{Método}

\section{Muestra}

La muestra estuvo compuesta de 120 estudiantes (50 chicos y 70 chicas) con edades comprendidas entre los 16 y 20 ańos $(M=16.73 ; D T=.74)$, pertenecientes primero de bachillerato 
de dos centros educativos españoles. La muestra fue dividida en un grupo experimental formado por 89 estudiantes (38 chicos y 51 chicas) y un grupo control constituido por 31 estudiantes (12 chicos y 19 chicas). El grupo control solo participó en la primera medida para asegurar que no existían diferencias previas con el grupo que luego se sometió a las tres situaciones experimentales.

\section{Medidas}

Frecuencia cardiaca. Se utilizaron pulsímetros de la marca POLAR y POLAR TEAM con los cuales se pretendía obtener la FC media de los estudiantes durante la práctica de ejercicio físico en la clase de educación física. Los pulsímetros utilizados fueron 8 del modelo "POLAR RS200", 2 del modelo "POLAR RS800” y 10 del "POLAR TEAM". En estos pulsímetros queda registrada la FC media y de esta manera se puede establecer en qué tipo de actividad física según intensidad se encuentran los estudiantes (ligera, moderada o vigorosa).

Vitalidad psicológica. Se utilizó el cuestionario Subjective vitality (Ryan y Frederick, 1997), adaptado al castellano por Molina-García, Castillo, y Pablos, (2007), para medir cómo se sentían los estudiantes en su bienestar situacional. Este cuestionario, cabe considerarlo como una medida eudaimónica del bienestar psicológico (Ryan et al., 2013). El cuestionario se compone de 7 ítems que indican cómo te sientes en el momento actual. Las respuestas son recogidas en una escala tipo Likert con un rango de puntuación que oscila desde 0 (no del todo cierto) a 7 (muy cierto). Los índices de fiabilidad en este estudio oscilaron entre .76 y .84 (Tabla 2)

Estado afectivo. Para representar los sentimientos positivos y negativos antes y después de la sesión de actividad física, se empleó el cuestionario de los nueve adjetivos utilizados en el Positive and Negative Affect Schedule (PANAS: Mackinnon et al. 1999; Watson, Clarck, y Tellegen, 1988). Este cuestionario se considera una medida hedónica del bienestar. La escala está compuesta por 9 adjetivos, cuatro de los cuales responden a sentimientos positivos "alegre, feliz, contento, divertido" y otros cinco, a sentimientos negativos "deprimido, preocupado, frustrado, enojado, infeliz". Las respuestas a dicha escala se responden en una escala tipo Likert que oscila desde 1 (no del todo) hasta 7 (extremadamente). Los índices de fiabilidad oscilaron entre .75 y .94 . Dado que no se ha validado el cuestionario al español, se realizó un AFC de dos factores, con la muestra inicial mostrando índices de ajuste adecuados. $\chi 2(118)=2,002, \mathrm{P}=.000 ; \mathrm{CFI}=.940 ; \mathrm{TLI}=.931$; $\mathrm{RMSEA}=.056$.

Calidad de sueño. Para medir la calidad de sueño de los estudiantes de educación física, se empleó la escala de sueño Karolinska Sleep Diary de Akerstedt, Hume, Minors, y Waterhouse, (1994). El diario se compone de siete ítems: "calidad de sueño, tranquilidad de sueño, facilidad para dormirme, despertar, facilidad para despertar, sensación de descanso y ¿has dormido lo suficiente?” Las respuestas son recogidas en una escala tipo Likert con un rango de puntuación que oscila desde 1 (muy pobre) a 5 (muy bueno) en el ítem correspondiente a "Calidad de sueńo, 1 (muy inquieto) a 5 (muy tranquilo) en el ítem "Tranquilidad de sueño", 1 (muy inquieto) a 5 (muy tranquilo) en el ítem "Facilidad para dormirme", 1 (desperté demasiado temprano) a 3 (no desperté temprano) en el ítem "Despertar", 1 (muy difícil) a 5 (muy fácil) en el ítem "Facilidad para despertar", 1 (no descansé en absoluto) a 3 (completamente descansado) en el ítem "Sensación de descanso" y de 1 (no, definitivamente muy poco) a 5 (Sí, definitivamente lo suficiente) en el ítem “¿Has dormido lo suficiente?”. Se calculó un AFC de un factor, obteniendo un valor de $\chi 2(32,118)=$ 2,002, $\mathrm{P}=.000 ; \mathrm{CFI}=.95 ; \mathrm{TLI}=.941 ; \mathrm{RMSEA}=.08$. El alpha de cronbach, osciló entre .66 y .85 en las diferentes situaciones experimentales

Motivación intrinseca situacional.: El factor "motivación intrínseca situacional” de la escala SIMS de Guay, Vallerand, y Blanchard, (2000) fue utilizado en su versión espańola (Martín-Albo, Núñez, y Navarro, 2009) para evaluar la motivación situacional en practicantes de actividad físicodeportiva. Dicha escala consta de 16 ítems, precedidos de la pregunta "¿en esta clase has practicado actividad física porque...?”, y se recogen las razones para practicar actividad física en una situación concreta. Nosotros sólo utilizamos el factor motivación intrínseca situacional, (p.e. "porque creo que esta actividad es agradable", "Porque esta actividad es divertida") dado que estudios previos (Texeira et al., 2012) relacionan la motivación intrínseca con la intensidad del ejercicio. Las respuestas a los ítems fueron reflejadas en una escala tipo Likert con un rango de respuesta de 1 a 7 , donde 1 correspondía a "no coincido en absoluto" y 7 a "coincido exactamente". El alpha de cronbach osciló entre .71 y .77.

\section{Procedimiento}

La muestra fue dividida en un grupo experimental formado por 89 estudiantes (38 chicos y 51 chicas) y un grupo control constituido por 31 estudiantes (12 chicos y 19 chicas). El grupo control solo participó en la toma inicial y nos sirvió para asegurar que el grupo que luego se sometió a las 3 situaciones experimentales, era homogéneo y no presentaba valores de referencia significativamente diferentes a la población. El manova calculado entre grupos, mostró que no existían diferencias significativas, por lo que podemos asegurar que los sujetos que participaron en el estudio, no diferían de la población a al que representaban (Lambda de Wilks $=.89 ; \mathrm{F}$ $(4,118)=.87 ; \mathrm{p}>.05)$

Se calculó la FC cardiaca máxima teórica en función de la fórmula de Tanaka (Tanaka, Monahan, y Seals, 2001), en 
la que se establece que la frecuencia cardíaca máxima de un sujeto se puede estimar a partir de la ecuación; FC max=208(0.7 x Edad), y se distribuyó aleatoriamente a los sujetos en función del porcentaje de FC media obtenido en cada sesión, respecto a su Frecuencia cardíaca máxima teórica, (ligera $<60 \%$, moderada $60-80 \%$, vigorosa $>80 \%$ ). Todos los sujetos del grupo experimental hicieron 3 sesiones, separadas entre ellas una semana, en la clase de educación física y con intensidad controlada. Para ello se efectuó un estudio piloto en el que se testaron las sesiones en un grupo de voluntarios, para asegurar que las diferencias de intensidad eran apreciables.

Una vez realizadas las sesiones, a cada sujeto se le clasificó en función de la intensidad relativa obtenida en cada sesión, a partir de la fórmula de Tanaka et al., (2001). Dado que el objetivo del trabajo era estudiar el efecto diferentes intensidades en ejercicio agudo sobre las variables comportamentales, aquellos sujetos que no alcanzaron el nivel de intensidad teórico en cada sesión fueron eliminados del análisis. No obstante, la muerte experimental por esta cuestión fue despreciable (12 casos). La parte principal de la sesión, tuvo una duración de 20 minutos, en la que se recogía la frecuencia cardiaca.

Se tomaron medidas de todas las variables previo a la realización de actividad física y al día siguiente (menos la motivación intrínseca situacional que se midió justo después de la realización de la sesión).

Además, en la primera medida, se tomaron antes de la intervención, medidas a un grupo control, para asegurar que los sujetos que se someterían a las tres situaciones experimentales no partían de medidas diferentes a las de la población objeto de estudio.

Todos los sujetos rellenaron un consentimiento informado, y aquellos que eran menores de edad presentaron, además, el consentimiento por parte de sus padres o tutores. Se les indicó que los datos obtenidos serían confidenciales y se les indicó que podían abandonar el estudio en cualquier momento, sin tener que dar ninguna explicación.

\section{Resultados}

\section{Análisis correlacional}

Para cumplimentar el primer objetivo del trabajo consistente en analizar las relaciones entre los diferentes tipos de bienestar psicológico se calcularon estadísticos descriptivos correlaciones bivariadas entre la vitalidad subjetiva, el estado afectivo positivo, el estado afectivo negativo y la calidad del sueño en las medidas pre intervención de todos los sujetos (experimental y control). Los resultados aparecen en la Tabla 1 y muestran que la vitalidad correlacionó de forma positiva y alta con el estado afectivo positivo, moderada y negativa con el estado afectivo negativo y de forma moderada y positiva con la calidad del sueño. A su vez, también el estado afectivo correlacionó con la calidad del sueño (positivamente el estado afectivo positivo y negativamente el estado afectivo negativo).

Tabla 1. Estadísticos descriptivos y correlaciones de las medidas pre-intervención entre la vitalidad, el estado afectivo y la calidad del sueńo, para toda la muestra.

\begin{tabular}{lllll}
\cline { 2 - 5 } & MD & 2 & 3 & 4 \\
\hline (1) Vitalidad & 4.90 & $.634^{* *}$ & $-.395^{* *}$ & $.338^{* *}$ \\
(2) Estado Afectivo Positivo & 5.22 & & $-.636^{* *}$ & $.371^{* *}$ \\
(3) Estado Afectivo Negativo & 2.14 & & & $-.361^{* *}$ \\
(4) Calidad del Sueño & 3.60 & & & \\
\hline
\end{tabular}

${ }^{* *} p<.01$

Análisis experimental

Se calcularon estadísticos descriptivos y pruebas de fiabilidad para todas las variables en la situación pre y en las tres situaciones experimentales (Tabla 2). Los resultados se presentan en el mismo orden en el que se realizaron las tres sesiones (moderada, vigorosa y ligera).

El MANOVA post-test, efectuado por grupo de intensidad, mostró un efecto significativo de la misma sobre algunas variables dependientes (Lambda de Wilks = .67, $F(10,126)=$ $1.71, p<.05)$. Concretamente, los ANOVAS posteriores, solo mostraron diferencias significativas entre las situaciones "actividad ligera" y "actividad vigorosa". Concretamente, encontramos diferencias significativas entre la situación ligera y vigorosa en el estado afectivo negativo $(F(2,87)=4.27 ; p<.05)$ y la motivación intrínseca situacional $(F(2,87)=3.85$; $p<.05)$, obteniendo un descenso significativo en estado afectivo negativo en la situación vigorosa $(M=1.3)$ frente a la ligera (1.7), y un mayor nivel de motivación intrínseca situacional post-sesión en la intensidad vigorosa $(M=5.7)$ frente a la ligera (4.3).

A continuación se calcularon pruebas " $\mathrm{t}$ " para muestras relacionadas en las 3 situaciones experimentales para comprobar el efecto que la práctica de ejercicio a diferentes intensidades tenía sobre el comportamiento del estado afectivo, la vitalidad y la calidad del sueño entre las situaciones pre y post práctica de actividad física.

Si analizamos la sesión de actividad física ligera, únicamente encontramos un incremento significativo en vitalidad subjetiva $(t=-4.91)$ entre la situación pre y post.

Por otra parte, al analizar los resultados de la actividad física moderada, los resultados muestran descensos significativos entre las medidas pre y post en los factores estado afectivo negativo $(t=$ 3.02) y curiosamente también en la calidad del sueńo $(t=-3.54)$.

Por último, al analizar la sesión de actividad física vigorosa, los resultados muestran incrementos significativos en vitalidad $(t=-3.17)$, estado afectivo positivo $(t=-3.04)$ y descensos en el estado afectivo negativo $(t=5.37)$, tras la sesión. 
Tabla 2. Descriptivos, fiabilidad y resultados de las pruebas t para muestras relacionadas en la situaciones pre y post de la frecuencia cardíaca media, bienestar subjetivo y motivación intrínseca situacional de las tres situaciones experimentales.

\begin{tabular}{|c|c|c|c|c|c|c|c|}
\hline \multirow{4}{*}{$\begin{array}{l}\text { Variables } \\
\text { Fc. Media }\end{array}$} & \multirow{4}{*}{$\begin{array}{l}\text { Alpha de Cronbach } \\
-- \\
\end{array}$} & \multicolumn{6}{|l|}{ MD } \\
\hline & & \multicolumn{2}{|c|}{ Ejercicio Moderado } & \multicolumn{2}{|c|}{ EjercicioVigoroso } & \multicolumn{2}{|c|}{ Ejercicio Ligero } \\
\hline & & \multicolumn{2}{|l|}{135} & \multicolumn{2}{|l|}{141} & \multicolumn{2}{|l|}{131} \\
\hline & & Pre & Post & Pre & Post & Pre & Post \\
\hline Vitalidad & $.76-.84$ & 5.10 & 5.00 & 4.95 & $5.33^{*}$ & 4.88 & $5.29^{*}$ \\
\hline Est. Afect. Positivo & $.81-.94$ & 5.42 & 5.62 & 5.36 & $5.74^{*}$ & 5.76 & 5.76 \\
\hline Est. Afect. Negativo & $.75-.87$ & 2.61 & $1.61^{*}$ & 1.76 & $1.32^{*}$ & 1.86 & 1.78 \\
\hline Calidad del Sueño & $.66-.85$ & 3.47 & $3.12^{*}$ & 3.22 & 3.16 & 3.28 & 3.24 \\
\hline Mot. Int. Situacional & $.71-.77$ & -- & 4.8 & -- & 5.7 & -- & 4.3 \\
\hline
\end{tabular}

\section{Discusión}

El objetivo de este estudio ha sido analizar, en primer lugar, la relación existente entre diferentes dimensiones del bienestar en clases de educación física de estudiantes adolescentes.

Nuestra primera hipótesis era que habría una relación positiva entre bienestar psicológico (vitalidad y estado afectivo positivo) y calidad del sueńo y negativa entre estado afectivo negativo y calidad del sueño.

Los resultados han mostrado, coherentemente con la hipótesis planteada, que tanto los componentes hedónicos como eudaimonicos del bienestar están estrechamente relacionados entre sí. Concretamente, hemos encontrado que la vitalidad subjetiva correlacionó de forma positiva y alta con el estado afectivo positivo y negativamente con el estado afectivo negativo. También hemos encontrado correlaciones entre los dos tipos de bienestar con la calidad del sueño, de forma que tanto la vitalidad como el estado afectivo positivo correlacionaron directamente con la calidad del sueño, mientras que el estado efectivo negativo lo hizo negativamente.

Estos resultados están en consonancia con los encontrados por Gunnel et al., (2014), que encontraron también correlaciones entre la vitalidad subjetiva y el estado afectivo positivo. Sin embargo, estos autores no encontraron relaciones entre vitalidad y estado afectivo negativo.

Otro resultado interesante del estudio correlacional, y que creemos es una aportación relevante del mismo, es la relación encontrada entre la calidad del sueño y las dimensiones del bienestar. Diferentes estudios han remarcado la importancia de la calidad del sueńo, como antecedente de una serie de problemas emocionales, físicos y psíquicos en diferentes poblaciones tanto de personas mayores (Polo- Kantola et al., 2014), como de adolescentes (Wong et al., 2013). En este último estudio, se encontró que la calidad del sueńo está relacionada con la salud, el rendimiento académico y, como en nuestro estudio, con el bienestar psicológico. A través de un modelo de ecuaciones estructurales, los investigadores encontraron relaciones directas e indirectas entre la cantidad y la calidad del sueño y el bienestar en adolescentes. Nosotros hemos indagado en estas relaciones y creemos que la calidad del sueño es una variable a incluir como aspecto relacionado con el bienestar. Futuras investigaciones deberían indagar en las relaciones existentes entre la calidad del sueño y otras variables de interés, por ejemplo la práctica de actividad física, como hemos comenzado a hacer en este trabajo.

Así, el segundo objetivo del trabajo fue examinar como la exposición a diferentes intensidades de ejercicio puede afectar al bienestar psicológico, la calidad del sueño y los niveles de motivación intrínseca situacional de los estudiantes. Nuestra segunda hipótesis era que esperábamos que la sesión de ejercicio vigorosa provocaría una mayor mejora del bienestar, calidad del sueño y motivación intrínseca situacional que las sesiones ligeras o moderadas y que todos los tipos de actividad física aguda provocarían cambios positivos en bienestar, y calidad del sueño frente a la situación de no actividad física.

A este respecto, hemos encontrado que, al comparar intensidades, aparecieron diferencias significativas post ejercicio, en las direcciones esperadas, entre las sesiones ligera y vigorosa tanto en motivación intrínseca situacional como en el estado afectivo negativo.

También queríamos analizar si la exposición a diferentes intensidades agudas de ejercicio ocasionaba cambios en el bienestar y la calidad del sueño, frente a la situación de no ejercicio. Encontramos que, efectivamente, la práctica de actividad física ocasionaba cambios en el bienestar y la calidad del sueńo. Concretamente, en el caso del bienestar, encontramos que a nivel general mejoraba el mismo, aunque solo encontramos diferencias significativas en vitalidad en la situación de intensidad vigorosa y ligera, mejoras del estado afectivo positivo en la situación vigorosa y descensos en el estado afectivo negativo en las situaciones moderada y vigorosa. Curiosamente, en todas las situaciones de ejercicio, 
independientemente de la intensidad empeoró la calidad del sueño, aunque solo la situación moderada mostró valores de descenso significativos.

Esos resultados muestran algunos aspectos interesantes sobre los que creemos se debe reflexionar. En primer lugar, parece ser que la práctica de actividad física vigorosa es la que afecta a más variables de las analizadas en el estudio, y además es la que se muestra como más adaptativa a la hora de provocar estos cambios, pues hemos visto que mejora la motivación intrínseca situacional cuando se compara con las otras intensidades, y además también mejora todas las dimensiones del bienestar cuando se compara entre situación de no práctica y práctica. Esto se muestra muy relevante puesto que, como comentamos en la introducción, los cambios psicológicos que se producen en el ejercicio están muy relacionados con la adherencia al mismo.

Si hablamos de las diferencias en motivación, hemos visto que ya hay estudios que analizan cómo diferentes tipos de motivación afectan a la práctica de actividad física. Así Texeira et al. (2012), encontró que las formas más autónomas de motivación predicen la cantidad de actividad física moderada y vigorosa que practican las personas que hacen actividad física habitualmente. Sin embargo, estos resultados se refieren al efecto de la motivación en la práctica crónica de ejercicio, mientras que nosotros hemos analizado el efecto de la práctica aguda en la motivación situacional. La idea es ver si diferentes intensidades afectan a la motivación, de forma que tengamos criterios claros para incluir la intensidad del ejercicio como una variable a tener en cuenta para incrementar la motivación. Desde el punto de vista práctico, nuestros resultados indican que es más adaptativo utilizar intensidades altas para provocar un incremento de la motivación intrínseca situacional si bien existe una limitación, referente a que la motivación situacional se puede haber visto afectada por el diseño mismo de la sesión (p.e. más divertida la sesión vigorosa que la moderada o la ligera), aunque se intentó cuidar que todas sesiones tuviesen contenidos parecidos. Creemos que se debe explorar más sobre este aspecto y profundizar en el efecto de la intensidad del ejercicio como variable que afecta a la motivación situacional, y porque no, contextual.

Por otra parte, al interpretar los datos de las pruebas " $\mathrm{t}$ " en la comparación de las situaciones de no práctica y práctica, existen ya trabajos previos que han mostrado que diferentes intensidades afectan de forma diferente a estados afectivos. Así, en un meta-análisis efectuado por Red y Ones (2006), se mostró que las intensidades moderadas y ligeras son las que provocan cambios afectivos más adaptativos desde el punto de vista del estado afectivo post sesión. Sin embargo, nosotros hemos encontrado que aunque las tres situaciones provocan cambios en alguna dimensión del bienestar, es la situación de intensidad vigorosa la única que provoca cambios en todas las dimensiones del mismo. Estos resultados parecen contradictorios con los trabajo previos, pero cabe destacar que nosotros hemos realizado un diseńo diferente en lo relativo al tiempo entre medida del bienestar y práctica de ejercicio. Nosotros medimos el bienestar el día mismo de realización del ejercicio, previo a la sesión, y el día después de efectuar el ejercicio, puesto que estábamos interesados en el efecto a medio plazo del ejercicio en el bienestar. La mayoría de estudios que han analizado el efecto del ejercicio agudo en el estado afectivo han efectuado medidas a corto plazo, puesto que parece ser que el efecto del mismo se disipa con el tiempo (Ekkekakis y Petruzello, 1999). Es por ello que es posible que la actividad física vigorosa sea la única que ha mostrado cambios en el bienestar en el día después, lo cual no quiere decir que el resto de intensidades no provoquen cambios (como así es en el caso de la vitalidad y actividad ligera o estado afectivo negativo en la actividad moderada), sino que es probable que este efecto se haya disipado con el tiempo. De todas formas, es interesante comprobar cómo el día siguiente a la realización del ejercicio, se siguen observando estos cambios. También desde una perspectiva práctica debemos tener en cuenta este aspecto puesto que si queremos incrementar la adherencia a la práctica de actividad física (sobre todo en personas sedentarias), quizás sea mas interesante provocar cambios en el bienestar lo suficientemente duraderos como para que las personas (fundamentalmente aquellas que son sedentarias), se sientan atraídas a repetir la realización de actividad física.

Si hablamos de la calidad del sueño, el comportamiento del mismo se ha alejado de lo esperado. Hemos encontrado que en todas las situaciones experimentales, la calidad del sueño disminuía. De hecho en la situación de actividad física moderada, este empeoramiento ha sido significativo.

Tal y como indican Wong et al. (2013), la calidad del sueño se está utilizando recientemente como un indicador de la salud en poblaciones clínicas, pero también en población no clínica. No conocemos muchos estudios que hayan analizado el tipo de actividad física y su efecto en la calidad del sueño, pero sí hemos encontrado trabajos (Akerstedt et al., 2002), que encontraron una relación directa entre la intensidad del esfuerzo ejercido en el trabajo y perturbaciones en el sueño. Por tanto, sí parece ser que la intensidad del ejercicio puede estar afectando al patrón de sueño. Una cuestión que no hemos analizado y que sería interesante explorar en el futuro es ver si estos resultados se ven afectados por el nivel de práctica de actividad física de los sujetos. Es posible que aquellas personas más adaptadas a la práctica de actividad física tengan más tolerancia y se adapten mejor al desequilibrio que supone la práctica de actividad física, y también se debería analizar si la calidad del sueño mejora tras la compensación fisiológica de este desequilibrio que supone la práctica de actividad física.

Como conclusión, comentar que la manipulación de la intensidad del ejercicio agudo parece ocasionar cambios en 
algunas dimensiones del bienestar de los adolescentes, pero debemos seguir indagando sobre los patrones de actividad física que son más adaptativos para mejorar todas las variables del bienestar.

Por último, comentar que este estudio tiene algunas limitaciones que creemos deben ser tenidas en cuenta en estudios futuros. En primer lugar se debería controlar el efecto que la práctica deportiva extraescolar tiene en las variables estudiadas. Se intentó controlar en la medida de lo posible, que los estudiantes no realizasen actividad física los días anteriores al experimento, pero es cierto que el patrón de sedentarismo o actividad física de cada uno de ellos es difícilmente controlable. Ésta es una variable que puede estar afectando a los resultados y deberíamos controlar. En segundo lugar la cuantificación de la intensidad de la sesión debe ser mejorada, pasando de estimaciones teóricas del nivel físico a estimaciones reales. Sería interesante efectuar una medición objetiva de la intensidad de la actividad física a través de acelerómetros e incluso comprobar hasta qué punto un patrón fuera de la escuela más o menos sedentario, podría estar influyendo en las variables estudiadas, con especial atención al comportamiento de la calidad del sueño.

\section{Aplicaciones prácticas}

A partir de los resultados encontrados en nuestro trabajo creemos que se derivan una serie de aplicaciones prácticas que es necesario destacar.

En primer lugar parece ser que diferentes intensidades de ejercicio, provocan diferentes consecuencias en la población objeto de estudio. Esto implica que a la hora de diseñar programas de intervención de actividad física, en los que queramos fomentar la adherencia a partir de la mejora de la vitalidad y el estado afectivo, la intensidad del ejercicio debe ser una variable a considerar. Los resultados muestran que las intensidades vigorosas son los que provocan mayores cambios, por lo que deberemos movernos alrededor de estas intensidades para obtener lo cambios más adaptativos. Otra cuestión importante hace referencia a la necesidad de evaluar los efectos psicológicos del ejercicio agudo. Si bien existen muchos trabajos que analizan el efecto crónico del ejercicio en el bienestar psicológico, es necesario que los psicólogos y los entrenadores presten más atención a los efectos agudos del ejercicio, puesto que hemos visto que ocasionan también cambios evaluables en el estado psicológico. Con todo ello es probable que acotemos de forma más clara el efecto que el ejercicio agudo tiene sobre el bienestar psicológico.

\section{Referencias}

1. Åkerstedt, T., Hume, K., Minors, D., y Waterhouse, J. (1994). The subjective meaning of good sleep, an intra-individual approach using the Karolinska Sleep Diary. Perceptual and Motor Skills, 79(1), 287296.

2. Åkerstedt, T., Knutsson, A., Westerholm, P., Theorell, T., Alfredsson, L., y Kecklund, G. (2002). Work organisation and unintentional sleep: results from the WOLF study. Occupational Environment Medicine, 59, 595-600

3. Deci, E. y Ryan, R. (2000). The "what" and "why" of goal pursuits: Human needs and the self-determination theory. Psychological Inquiry, 11, 227-268.

4. Ekkekakis, P., y Petruzzello, S.J. (1999). Acute aerobic exercise and affect: Current status, problems, and prospects regarding dose-response. Sports Medicine, 28, 337-374.

5. Eurobarómetro (2013). European Commission, Directorate-General for Education and Culture. http://ec.europa.eu/public_opinion/archives/eb/eb79/eb79_en.htm.

6. Guay, F., Vallerand, R., y Blanchard, C. (2000). On the assessment of state intrinsic and extrinsic motivation: The situational motivation scale (SIMS). Motivation and Emotion, 24, 175-213

7. Gunnel, K., Crocker, P., Mack, D., Wilson, P., y Zumbo, B. (2014). Goal contents, motivation, psychological need satisfaction, well-being and physical activity: A test of self-determination theory over 6 months. Psychology of Sport and Exercise, 15, 19-29.

8. Martín-Albo, J., Núnez, J. L., y Navarro, J. G. (2009). Validation of the Spanish version of the Situational Motivation Scale (EMSI) in the educational context. Spanish Journal of Psychology, 12, 799-807.

9. Mackinnon, A., Jorm, A. F., Christensen, H., Korten, A. E., Jacomb, P. A., y Rodgers, B. (1999). A short form of the positive and negative affect schedule: evaluation of factorial validity and invariance across demo- graphic variables in a community sample. Personality and Individual Differences, 27, 405-416.

10. Molina-García, J., Castillo, I., y Pablos, C. (2007). Bienestar psicológico y práctica deportiva en universitarios. Motricidad. European Journal of Human Movement, 18, 79-91.

11. Moreno, J. A., Gómez, A. y Cervelló, E. (2010). Un estudio del efecto de la cesión de autonomía en la motivación sobre las clases de educación física. Motricidad. European Journal of Human Movement, 24, 1-21.

12. Polo-Kantola, P., Laine, A., Aromaa, M., Rautava, P, Markkula, J., Vahlberg, T., Sillanpaa, M. (2014). A population-based survey of sleep disturbances in middle-aged women - Associations with health, health related quality of life and health behavior. Maturitas, 77, 255-262.

13. Reed, J., y Ones, D. (2006). The effect of acute aerobic exercise on positive activated affect: A meta-analysis. Psychology of Sport and Exercise, 7, 477-514.

14. Ryan, R. y Deci, E. (2000). Self-determination theory and the facilitation of intrinsic motivation, social development and well-being. American Psychologist, 55, 68-78.

15. Ryan, R. y Frederik C. (1997). On Energy, Personality, and Health: Subjective Vitality as a Dynamic Reflection of Well-Being. Journal of Personality, 65, 529-565.

16. Ryan, R., Huta, V., y Deci, E. (2008). Living well: a self-determination perspective on eudaumonia. Journal of Happiness Studies, 9, 139-170.

17. Ryan, R., Huta, V., y Deci, E. (2013). Living well: a self-determination perspective on eudaumonic. En A. Delle-Fave (Ed.), The exploration of happiness, happiness studies book series (pp 117-139). The Netherlands: Springer.

18. Tanaka, H., Monahan, K., y Seals, D. (2001). Age-predicted maximal heart rate revisted. Journal of the American College of Cardiology, 37,153156. 
19. Texeira, P., Carraça, E., Markland, D., Silva, M., y Ryan, R. (2012). Exercise, physical activity, and self-determination theory: a systematic review. International Journal of Behavioral Nutrition and Physical Activity, 9-78.

20. Thayer, R. (1996). The origin of everyday moods: managing energy, tension and stress. New York: Oxford University Press.

21. Vallerand, R. J. (1997). Toward a hierarchical model of intrinsic and extrinsic motivation. In M. P. Zanna (Ed.), Advances in experimental social psychology (pp. 271-360). New York: Academic Press.

22. Vallerand, R.J. (2001). A hierarchical model of intrinsic and extrinsic motivation in sport and exercise. En G.C. Roberts (Ed.), Advances in motivation in sport and exercise (pp. 263-320). Champaign, IL: Human Kinetics.

23. Vallerand, R. J. (2007) Intrinsic and Extrinsic Motivation in Sport and Physical Activity: A Review and a Look at the Future, in Handbook of
Sport Psychology, (eds G. Tenenbaum and R. C. Eklund), John Wiley \& Sons, Inc., Hoboken, NJ.

24. Vazquez, C., Hervás, G., Rahona, J., y Gómez, D. (2009). Bienestar psicológico y salud: Aportaciones desde la psicología positiva. Anuario de Psicología Clínica y de la Salud, 5, 15-28.

25. Viladrich, C., Torregrosa, M., y Cruz, J. (2011). Calidad psicométrica de la adaptación espańola del Cuestionario de Regulación Conductual en el Deporte. Psicothema, 23, 786-794.

26. Watson, D., Clark, L. A., \& Tellegen, A. (1988). Development and validation of brief measures of positive and negative affect: the PANAS scales. Journal of Personality and Social Psychology, 54, 1063-1070.

27. Wong, M., Lau, E., Wan, J., Cheung, S., Hui, C., y MOK, D. (2013) The interplay between sleep and mood in predicting academic functioning, physical health and psychological health: A longitudinal study. Journal of Psychosomatic Research, 74, 4, 271-277. 